\title{
What assistive technologies exist to enable participation in play?
}

\author{
Pedro Encarnação and Rianne Jansens
}

Participation in play often requires staying in a comfortable and functional position, moving around, manipulating objects, communicating, or using a computer or tablet. This chapter briefly refers to technologies that may reduce the gap between the requirements of the play and the children's abilities by providing support in the above general-purpose functional areas or by adapting the activity. Adapted toys and games are discussed in Chapter 7 and accessible digital games in Chapter 8.

\section{What is Assistive Technology?}

Following the World Health Organization definition, Assistive Technology 'is the application of organized knowledge and skills related to assistive products, including systems and services'. An Assistive Product is 'any external product (including devices, equipment, instruments or software), especially produced or generally available, the primary purpose of which is to maintain or improve an individual's functioning and independence, and thereby promote their well-being'.

The International Organization for Standardization (ISO) issued a classification of assistive products in its ISO 9999 international standard. This classification is widely used around the globe, especially for prescription of assistive products purposes. An overview of the different ISO 9999 classes can be obtained at the web page http://www.eastin.eu/en/searches/products/iso maintained by EASTIN. EASTIN is the European Assistive Technology Information Network offering information on assistive technology to all citizens. The network features the website http://www.eastin.eu where one can find, in all official languages of the European Union, and in a user-friendly and accessible manner, information 
and guidance tools on assistive technology. Over 70.000 assistive products (APs) are covered including practical information on assistive solutions for daily living problems.

Assistive products may be custom-made, satisfying the particular needs of a particular user (e.g. a custom contoured cushion); may be commercially available, having been developed for a generic user with a given condition (e.g. a wheelchair); or can be a mainstream technology developed for all (e.g. a computer or even Velcro ${ }^{\circledR}$ when used to help object manipulation). In reality, assistive products may lie in a continuum from custom-made to mainstream technology. For example, one can adapt a commercially available AP so it fits the particular needs of a user, thus lying between a commercially available and a custom-made assistive product. Mainstream assistive products are typically easier to obtain and less expensive than devices that are produced specifically for individuals with disabilities. However, it can be harder to obtain funding for mainstream products since funding programs often exclude those products to prevent abuse.

\section{APs for seating and positioning}

If not in a comfortable position that promotes function, a child is not able to play or interact. APs for seating and positioning serve three goals: postural control (ISO Code 09.07), tissue integrity (ISO Code 04.33), and comfort. Postural control is needed when children have impaired motor control such as abnormal muscle tone, muscle weakness, primitive reflexes or uncoordinated movements. Children that use a wheelchair and have limited ability to reposition themselves and/or do not sense pain or discomfort are at a risk of developing pressure ulcers, thus may need some assistance for keeping tissue integrity. For some children with mobility impairments, sitting for a long time causes discomfort from which they are unable to obtain relief. This discomfort may decrease their ability to participate in play. Technologies used to address the three goals above include seating systems like cushions, available in different materials with different properties, posture supports, belts, harnesses or straps to restrain uncontrolled movements or support the trunk and limbs for enabling fine motor movements. Postural control may also be facilitated with specific purpose benches or chairs, with non-standard dimensions and/or support components (e.g. head, arm, hip, or leg rests). 
Children play in all kind of positions: standing, seated on the floor or on a chair, lying on their bellies or on their backs, knelt or crouched down, hanging from a sofa or bed, etc. They often change position while playing depending on the experienced comfort and in response to the demands of the task in relation to their abilities. This constitutes a challenge when considering seating and positioning APs to enable participation in play and frequently non-traditional solutions need to be adopted, along with adaptations to the playspace, in order not to spoil the play experience.

\section{APs for mobility}

Many times playing requires functional mobility, or participation is enhanced by being able to move independently. Children with mobility impairments may need only a cane, walker or crutch to support mobility (ISO Codes 12.03 and 12.06), but for more severe impairments, a wheelchair, manual (ISO Code 12.22) or powered (ISO Code 12.23), may be necessary. For outdoor play, pedal-propelled vehicles for two or more persons (ISO Code 12.18.15) can create play opportunities (e.g. enabling participation in a hide and seek game).

In the past, several concerns advised against the use of powered mobility by young children, including safety issues, acquisition and replacement costs, and possible detrimental effects on the development of self-locomotion. However, research in the last years supports the provision of powered mobility to children at an appropriate developmental time (the closest to when typically developing children start to walk as possible). Research also shows that training children on the use of a wheelchair should not be focused on learning how to control the wheelchair but rather on achieving functional goals (e.g. participating in a play-like activity) using the wheelchair.

\section{APs for manipulation}

Often play involves the manipulation of objects (e.g. toys), which may be challenging for children with physical impairments. APs for manipulation range from low-tech devices that facilitate grasping (ISO Code 24.18.03), reaching (ISO Code 24.21.03), or holding (ISO Code 24.18.09), to high-tech devices such as robotic manipulators (ISO Code 24.18.30) or prosthesis (ISO Code 06.18.24). Today, with 3D printing, it 
is possible to obtain an inexpensive functional prosthesis. Examples can be found in the platform Patient Innovation (https://patient-innovation.com/), a platform that shares solutions created by patients (or their carers) to help them coping with their day-to-day challenges. Lego ${ }^{\circledR}$ Mindstorms ${ }^{\circledR}$ robots (https://www.lego.com/ en-us/mindstorms/) have also been used as manipulation tools enabling children to participate in play activities. Manipulation also includes operating and controlling devices. Push buttons (ISO Code 24.09.03) or switches (ISO Code 24.09.18) are examples of APs that may enable a child to control an electronic toy.

\section{APs for communication}

Communication is the very essence of being human and to be able to communicate with others is required for full participation in play, either to interact with playmates or with a play facilitator. Children with complex communication needs may use speech-generating devices (ISO Code 22.21.09) to produce voice output from a written text or by selecting an appropriate option in an augmentative and alternative communication (AAC) software.

Nowadays AAC software is available for mainstream technology such as iPads or smart phones. These might be preferable for children, provided that the child has the necessary fine motor and cognitive skills to use them, since the stigma associated to these devices is smaller. Communication via an AAC software is often slow, requiring the user to write the text to be spoken or to go through a set of selections. In play situations, this delay can hamper participation. Simple devices as switches that produce digitally recorded speech output upon a switch press may be a good alternative for some play scenarios (e.g. telling a story together where each child is responsible for the voice of a character; the character lines may be pre-recorded and the child only has to hit the switch when it is his/her turn). Other concerns when using AAC devices in play include making sure that appropriate vocabulary is available for the child and that the AAC system is mounted such that it does not get in the way of play.

Another way of addressing communication issues in play is to incorporate different forms of communication within the play objects. For example, parts of a toy/game can have different textures or contain braille text; tactile feedback may be provided in addition to auditory and/or visual feedback; visual information can include pictures, pictorial communication symbols, or sign language. 


\section{APs for accessing the computer or tablet}

When using a computer, one has to provide inputs using the keyboard, a mouse, a touch screen, or another input system. That can be done by using a method to select directly the desired option among all the possible options. For example, one can directly select one letter in a computer keyboard, or use a mouse or a finger to directly select an option on a screen, or use the voice to indicate the choice. Direct selection is the fastest and easier selection method but requires refined and controlled movements. Input devices for direct selection (ISO Code 22.36) include keyboards (standard or modified), mice (standard or modified), eye tracking systems, or head pointers. When direct selection is impossible, the different options can be presented one at a time to the user that can select the desired with a single movement (e.g. pressing a switch). This is called indirect selection through scanning. It requires very little motor control but good visual tracking skills, a high degree of attention, and the ability to sequence. There is a great variety of switches (ISO Code 24.09.18) to support indirect selection (e.g. mechanical, electromagnetic, electrical control, proximity, pneumatic, or phonation switches). Special care should be given to the positioning of the input devices and routing of the cables, maximizing function and not interfering with the activity.

\section{APs for seeing and hearing}

Visual and auditory impairments may constitute a barrier to play. Anyone watching children playing will observe that visual information and sounds are an important part of any activity. Children with low vision can benefit from APs that augment their visual abilities such as lenses (ISO Code 22.03.09) or devices for displaying an enlarged image of the subject that has been captured by a video camera (ISO code 22.03.18). For blind persons, APs can provide alternative ways to convey visual information, translating visual inputs to tactile or auditory inputs. Examples are tactile computer displays (ISO Code 22.39.05) or screen readers (22.39.12).

Devices for concentrating, amplifying and modulating sound (ISO Code 22.06) may augment the hearing abilities of children with hearing loss. Voice to text software 
(ISO Code 22.36.18) or sound indicators (ISO Code 22.27.18) provide alternative sensory pathways for deaf children that can be used in play situations.

\section{APs for understanding the play situation}

Sometimes play involves following rules, often set on the fly by playmates. When children play with toys, usually they attribute them different meanings and functions, varying over time. This can be challenging for a child with cognitive impairments. APs can help to keep track of instructions or time (ISO Codes 22.27.12, 22.27.15, 22.27.16 and 22.27.33). APs for marking and identifying materials (ISO Code 22.27.27) can be used to make clear different meanings of an object.

AAC products for face-to-face communication (ISO Code 22.21) and AAC techniques may be helpful to provide instructions about the play in an easy to understand way or to provide an overview of the activity that the child can refer back to when needed.

Play can involve many partners, be very dynamic and noisy, overwhelming children with different stimuli. A headphone (ISO Code 22.06.06) can help a child to focus and reduce stimuli.

\section{Design for All}

According to the Design for All Foundation (http://designforall.org/), 'Design for All [Universal Design] is the intervention into environments, products and services which aims to ensure that anyone, including future generations, regardless of age, gender, capacities or cultural background, can participate in social, economic, cultural and leisure activities with equal opportunities'. Design for All fosters user-centred design where the diverse needs of all are taken into account. A day-to-day example is elevator button panels with braille labels. The major computer, tablets and mobile phones operating systems have already built in accessibility functions. They include high contrast themes, a narrator that reads aloud any text on screen, 
a magnifier that zooms the area of the screen where the mouse is, changing the mouse cursor size and speed, on-screen keyboards, and voice-control. These are available through 'Ease of Access Centre' in Windows, 'Accessibility' options in iOS and Android. In some cases, they may be enough to make the device accessible for a particular child, thus avoiding the need of additional assistive technology.

\section{The process of choosing an AP}

Choosing an Assistive Product should be a user-centred process. It is the user, with his/her physical, cognitive and emotional characteristics, that wants to perform an activity, within a given physical, social, cultural and institutional context, that should have the final word in the choice of an AP to support him/her. This is also true for children, especially because their viewpoints on activities and assistive technology are often different from those of adults. For example, an AP might be fun and interesting to a child, but the stigma of its use may make it difficult for a parent to accept it for the child. Assessment for assistive technology should be made by a multidisciplinary team and be as comprehensive as possible, evaluating not only the needs of the particular client but also the context in which the AP will be used. Family members and significant others should be involved in the process since many times they will be co-users of the AP.

\section{APs funding}

In Europe, each country's social insurance or tax system finance assistive technology. Often public funding of assistive technology is described in relevant social legislation. For example, in some countries, lists of assistive products that are financed by public funds are published. It is worth mentioning that all European countries have signed the United Nations Convention on the Right of Persons with Disabilities and, with the exception of Ireland and Belarus, the Convention was already ratified by the national parliaments. Article 4 of this Convention forces the signatories to perform and promote research and development in assistive technology and design for all, and to make available accessible information on assistive technology. The great majority of the European countries also ratified the Optional Protocol to the Convention, enabling citizens to appeal to the Committee 
on the Rights of Persons with Disabilities when they believe they are victims of a violation by that country of the provisions of the Convention. Private insurances may also cover assistive technology expenses. Clinicians are aware of the requirements and of the process to apply for public and private funding and thus can assist the client in obtaining funding for assistive technology.

\section{Conclusion}

Play is a very diverse activity. Children play on their own or with others, with or without objects. Their play may be boisterous or quiet, light-hearted or very serious. By definition, play is an activity for enjoyment and recreation, without a practical purpose, which can make it hard to understand for those not participating in it. Therefore, requirements of play can also be very diverse. They may require a child to stand still quiet or to move around; to communicate with others or to understand and follow instructions; to manipulate objects or access a computer.

There are many Assistive Products than can enable participation of a child with disabilities in play, either by supporting children in some functional task, or by adapting the task such that it becomes accessible to a particular child. When selecting an AP to support play, the characteristics of the play need to be taken into consideration such that the AP does not come in the way of fun and enjoyment. Take your time exploring the EASTIN database for choosing an AP. The ISO Codes provided in this chapter may guide your search. Many times one needs to think out of the box, adapting APs that were mainly developed for other purposes. Be creative!

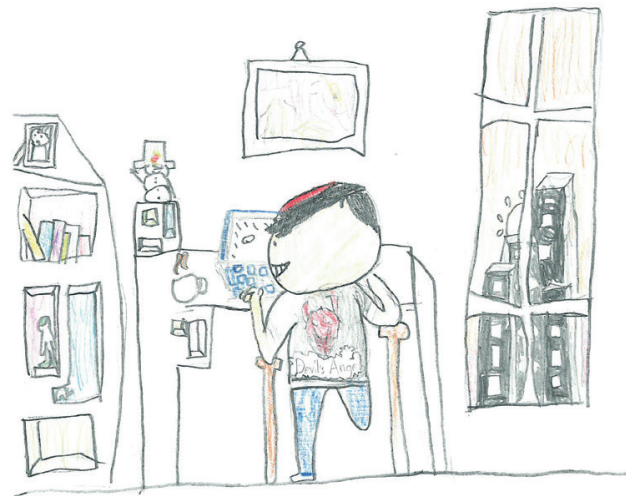

Daniel, 10 years old, Malta

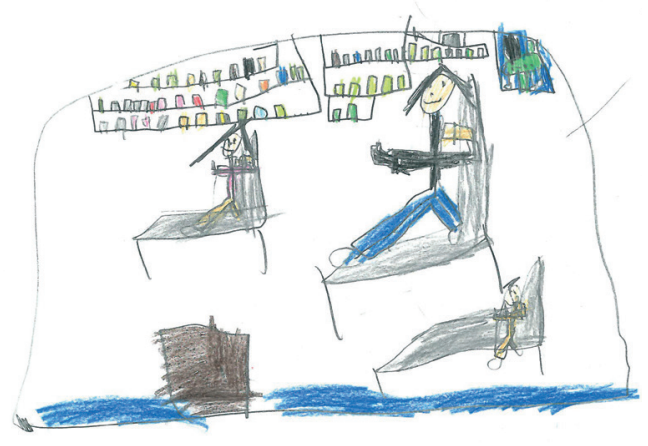

Aiden, 6 years old, Malta 